\title{
Sigmoid Type Neuromorphic Activation Function Based on Saturable Absorption Behavior of Graphene/PMMA Composite for Intensity Modulation of Surface Plasmon Polariton signals
}

Hamed Tari ( $\square$ hamed.Tari@sbai.uniroma1.it)

Sapienza University of Rome: Universita degli Studi di Roma La Sapienza https://orcid.org/0000-00017724-4538

\section{Alessandro Bile}

Sapienza University of Rome: Universita degli Studi di Roma La Sapienza

Francesca Moratti

Sapienza University of Rome: Universita degli Studi di Roma La Sapienza

\section{Eugenio Fazio}

Sapienza University of Rome: Universita degli Studi di Roma La Sapienza

\section{Original Article}

Keywords: Neuromorphic activation function, Surface Plasmon Polariton, photonic synapse, Saturable absorber

Posted Date: February 8th, 2021

DOI: https://doi.org/10.21203/rs.3.rs-170869/v1

License: (c) (1) This work is licensed under a Creative Commons Attribution 4.0 International License.

Read Full License

Version of Record: A version of this preprint was published at Plasmonics on January 21st, 2022. See the published version at https://doi.org/10.1007/s11468-021-01553-z. 
Hamed Tari ${ }^{1}$, Alessandro Bile, Francesca Moratti, Eugenio Fazio

\title{
Emails:
}

- Hamed.tari@sbai.uniroma1.it

- Alessandro.bile@uniroma1.it

- Francesca.moratti3@gmail.com

- Eugenio.fazio@uniroma1.it

\section{Sigmoid type neuromorphic activation function based on saturable absorption behavior of Graphene/PMMA composite for intensity modulation of Surface Plasmon Polariton signals}

\begin{abstract}
:
For an optical technology to be feasible as a substitution of electronics one, it is required the waveguides be capable of confining and directing light signals in much smaller dimensions than the operating wavelength of the light, i.e. subwavelength optical components must be realized. One approach to miniaturize the size of the photonic components is to benefit the hybrid nature of Surface Plasmon Polariton which addresses the problem with the diffraction limit of regular photonic components. Here, the authors present a two-dimensional numerical simulation of a passive photonic element based on a saturable absorber material as an analogy to the biological neurons activation function. They demonstrate that at telecom wavelengths a highly confined SPP mode can be modulated in a nonlinear fashion by considering the carrier dynamics of graphene/PMMA polymeric composite as a twolevel system. The sigmoid type nonlinear activation function derived from this study, further characterized and the parameters which appeared to be effective on the performance of the structure, such as saturation intensity, modulation depth, and thickness of the synaptic part as a Fabry-Perot structure, has been studied.
\end{abstract}

Keywords: Neuromorphic activation function; Surface Plasmon Polariton; photonic synapse; Saturable absorber

\section{Introduction}

In recent years, one-dimensional (1D) nanowires or nanofibers, with diameters of tens to hundreds of nanometers and lengths up to tens of millimeters, have been attracting increasing interests for photonic applications [1]. Generally, photonic nanowires, such as semiconductor nanowires [2], nonlinear optical crystals waveguide [3], glass nanofibers [4] or silicon-on-insulator waveguides [5], exhibit low optical waveguiding losses, but their optical confinement is limited by the diffraction limit that is on the order of $\lambda / n$ (with $n<5$ ). In contrast, by coupling light to the collective oscillations of electron on the noble metal interface and a dielectric layer, the electromagnetic field of the photons can be trapped on the surface due to its interaction with the free conduction electrons of the metal. The hybrid nature of SPP makes it possible to simultaneously carry optical and electrical signals in the same circuitry and interact properly with the electronic and photonic components. Moreover, the high localization and surface confinement of Surface Plasmon Polariton (SPP), greatly enhances the intensity at the 2-dimensional (2D). This hybrid characteristic of the SPP collects the advantages of photonic (such as higher bandwidth and higher speed) and electronic technology (the higher interaction and lower size and wavelength) and enables the design of the computationally fast and highly dense neural network.

\footnotetext{
${ }^{1}$ Corresponding author: Hamed Tari

Department of Basic and Applied Sciences for Engineering, Sapienza University of Roma, 00161 Roma ITALY
} 
Unrestricted by the diffraction limit, plasmonics structures can enable stronger modal confinement to enhance optical processes and miniaturize photonic circuits. Though, the electromagnetic waves at telecom frequency can be tightly confined along with the interface of a metal and a dielectric layer, and guide the light beyond the diffraction limit in a metal nano strip [6]. However, despite that the wider metal stripes can effectively guide the SPP, nevertheless, when the width of the stripe becomes smaller, the losses significantly increase due to scattering on the stripe edges, preventing scaling down such waveguides. In order to overcome this problem, dielectric waveguides for SPPs on a metal surface have been recently proposed [7]. The dielectric results in a higher refractive index for an SPP wave on a metal-dielectric interface compared to the metal-air interface, giving rise to SPP modes bound by the dielectric stripe similar to the guided light modes in conventional optical fibers or planar dielectric waveguides. Thus, stronger confinement and reduction of edge-scattering losses in dielectric-loaded SPP waveguides (DLSPPWs) can be achieved compared to bare metallic stripe waveguides of the same width. Moreover, a dielectric coating can be functionalized to provide thermo, electro, or all-optical functionalities and can be used for the development of active and passive modulation of plasmonics components.

Compared to the active SPP modulation, the passive modulation of SPP which is based on the nonlinear interaction of the material to the light, has advantages in compactness, lower complexity of integration, low cost, etc. The Kerr effect and saturable absorption are the main phenomena used in passive optical modulators, both of which represent third-order optical nonlinearities, where the former is determined by the real part of the optical susceptibility and the latter is related to the imaginary part. Compared with the Kerr effect, saturable absorption is a modulation of the absorption and has advantages in some aspects, such as tunable and large modulation depth. However, along the generated losses during saturable absorption process which is determined by the sensitivity of the absorption to light intensity, the indirect $1.12 \mathrm{eV}$ band-gap of silicon limits its application in passive modulators, especially at telecommunications wavelengths e.g. $1.3(0.95 \mathrm{eV})$ and $1.5 \mu \mathrm{m}(0.8 \mathrm{eV})$ [8]. Bao et al. [9] have recently reported that graphene can provide outstanding saturable absorption, overcoming these inherent drawbacks of semiconductor saturable absorber materials. Because of its unique energy band properties with the Pauli blocking principle, graphene possesses significant advantages, including ultra-low saturation intensity, larger saturable-absorption modulation depth, higher damage threshold, ultrafast recovery time, and a broad wavelengthindependent saturable absorption range $(300-2500 \mathrm{~nm})[10]$.

Here, what the study intends to accomplish is to numerically simulate a photonic neuron based on SPP for neuromorphic application purposes. The neuron consists of a saturable absorber that is responsible for the nonlinear transmission of the SPP. The saturable absorber defines a threshold value for the transmission of incoming SPP field and as a result, its intensity undergoes a passive and nonlinear modulation. It has been demonstrated that at telecom wavelengths a highly confined SPP mode can be modulated in a nonlinear fashion by considering the carrier dynamics of graphene/PMMA polymeric composite as a two-level system. The saturable absorber behavior of the graphene/PMMA results to a sigmoid type non-linear activation function and the parameters which appeared to be effective on the performance of the neuron, such as saturation intensity, modulation depth, and thickness of the saturable absorber has been optimized accordingly.

\section{Simulation method}

Since the Plasmon effect is essentially a near field phenomenon, only near field in this simulation is calculated. We have started with 2D simulations in order to be able to use extra fine mesh for higher preciseness. All domain boundaries except the bottom of the Ag strip are surrounded by Perfectly Matched Layer (PML) to absorb outgoing waves. The skin depth of Ag at visible wavelength is about $20 \mathrm{~nm}$, so the transmission through $100 \mathrm{~nm} \mathrm{Ag} \mathrm{film} \mathrm{is}$ negligible, and no PML is needed at the bottom boundary. PML at the top boundary assumes infinite half space for $\mathrm{SiO} 2$ interface. Note that special care needs to be taken for PML set up at the metal/dielectric interface region for appropriate absorption of the SPP waves. It has to be noted, at higher values of the input power still a small portion of the light reflects back inside the propagation area and in order to prevent their interference with the SPP, we have considered the outermost boundary of the PML as second order scattering boundary condition which ensures nearly zero back reflection of the light inside the propagation area.

The optical properties of the composite material (graphene/PMMA) such as saturable and nonsaturable absorption coefficient, photo induced electron- hole density and carrier recombination time has been tabulated for interpolation. A periodic port, has been utilized to illuminate the structure at the grating region to couple the light 
to SPP. Figure 1, represents the schematic view of the simulated structure with $1 \mathrm{~mW}$ input power. The SPP propagates along the metal/dielectric interface and shows a great surface confinement. Since the intensity of the received SPP is lower than the saturation intensity of the saturable absorber, so the SPP field is completely suppressed and no SPP is able to be transmitted to the post synaptic region.

\section{(B)}

Figure 1(A) Schematic representation of the simulated structure (B) Excitation of the structure with a periodic port with intensity before saturation intensity of the saturable absorber and complete suppression of the SPP field.

\section{Effect of different dielectric materials on SPP propagation}

The important parameter which has been analyzed here, is the material sweep for studying the effect of different dielectric materials to the SPP propagation. The selected materials in this simulation have been taken from the most popular materials in the semiconductor industry which all of them are CMOS compatible and fabrication of them is commercially available. Table.1, summarizes the calculated parameters for different materials. According to these data, it is possible to vary the SPP wavelength simply by changing the dielectric material and cover a broad range of wavelengths. Taking into account the imaginary part of the propagation constant, it is possible to calculate the length $L_{S P P}=1 / 2 k_{S P P}^{\prime \prime}$ in which the SPP field intensity can propagate along the interface with a decay factor of $1 / \mathrm{e}$ by considering the $k_{S P P}^{\prime \prime}$ parallel to the interface as follow

$$
\begin{aligned}
& k_{S P P, x}^{\prime \prime} \approx \frac{\omega}{c} \sqrt{\frac{\varepsilon_{1}^{\prime} \varepsilon_{2}}{\varepsilon_{1}^{\prime}+\varepsilon_{2}}} \frac{\varepsilon_{1}^{\prime \prime} \varepsilon_{2}}{2 \varepsilon_{1}^{\prime}\left(\varepsilon_{1}^{\prime}+\varepsilon_{2}\right)} \\
& k_{1, z}=\frac{\omega}{c} \sqrt{\frac{\left(\varepsilon_{1}^{\prime}\right)^{2}}{\varepsilon_{1}^{\prime}+\varepsilon_{2}}}\left[1+i \frac{\varepsilon_{1}^{\prime \prime}}{2 \varepsilon_{1}^{\prime}}\right] \\
& k_{2, z}=\frac{\omega}{c} \sqrt{\frac{\left(\varepsilon_{1}^{\prime}\right)^{2}}{\varepsilon_{1}^{\prime}+\varepsilon_{2}}}\left[1-i \frac{\varepsilon_{1}^{\prime \prime}}{2\left(\varepsilon_{1}^{\prime}+\varepsilon_{2}\right)}\right]
\end{aligned}
$$

where the $\varepsilon_{1}$ and $\varepsilon_{2}$ is the permittivity of the metal and dielectric layer respectively. From the collected data for different dielectric materials, it is clear that, to have a long propagation length along the interface and penetration depth of SPP normal to the interface, it is required to implement a dielectric material with a lower refractive index. The highest propagation length, belongs to the $\mathrm{SiO} 2$ which its lower refractive index allows the SPP to propagate about $400 \mu \mathrm{m}$ along with the interface. The lowest value for SPP propagation length is for InGaAs, which no matter how much the angle of incidence is, the propagation length barely reaches to $5 \mu \mathrm{m}$. To calculate how much SPP is able to penetrate inside the metallic layer $\left(\delta_{\mathrm{m}}\right)$ and the dielectric layer $\left(\delta_{\mathrm{d}}\right)$, we have implemented Equation (2) and (3) respectively. The electric field penetration depth of SPP for metal and dielectric layer is $1 / k_{1, z}$ and $1 / k_{2, z}$ respectively. The penetration depth for the metallic layer is almost the same for all of the materials but for the dielectric materials changes in a broad range. As a result, for a more confined SPP field inside the dielectric layer, one can use a dielectric material with a higher refractive index. From the obtained results, the highest value of field penetration inside the dielectric layer belongs to the $\mathrm{SiO}_{2}$ and the lowest value belongs to $\mathrm{Si}$ and $\mathrm{InGaAs}$. 
Table.1: Different material optical properties and SPP propagation parameters: SPP wavelength, propagation length for decay of intensity of SPP by $1 / \mathrm{e}$, penetration depth of SPP in metal layer, penetration depth in dielectric layer, normalized intensity for SPP received at the end of structure and finally the grating wavelength (groove width) respectively.

\begin{tabular}{llllllll}
\hline Materials & $\mathrm{n}$ & $\begin{array}{l}\lambda_{\mathrm{SPP}} \\
(\mu \mathrm{m})\end{array}$ & $\mathrm{L}_{\mathrm{SPP}}(\mu \mathrm{m})$ & $\begin{array}{l}\delta_{\mathrm{m}} \\
(\mathrm{nm})\end{array}$ & $\begin{array}{l}\delta_{\mathrm{d}} \\
(\mu \mathrm{m})\end{array}$ & $\begin{array}{l}\mathrm{I}_{\mathrm{SPP}} \\
\left(\mathrm{W} / \mathrm{cm}^{2}\right)\end{array}$ & $\begin{array}{l}\lambda_{\mathrm{g}} \\
(\mu \mathrm{m})\end{array}$ \\
\hline $\mathrm{SiO}_{2}$ & 1.444 & 1.0734 & 406 & 21.53 & 1.334 & 1.9144 & 1.420 \\
Photoresist & 1.575 & 0.9834 & 311 & 21.49 & 1.119 & 1.2912 & 1343 \\
$\mathrm{AIN} \mathrm{Al}_{2} \mathrm{O}_{3}$ & 1.765 & 0.8781 & 220 & 21.44 & 0.889 & 0.9294 & 1.246 \\
$\mathrm{Al}_{\mathrm{x}} \mathrm{Ga}_{1-\mathrm{x}} \mathrm{As}$ & 3.243 & 0.4776 & 32 & 20.8 & 0.255 & 0.0589 & 0.795 \\
$\mathrm{In}_{\mathrm{x}} \mathrm{Ga}_{1 \times} \mathrm{As}{ }_{1-\mathrm{y}} \mathrm{P}_{\mathrm{y}}$ & 3.248 & 0.4767 & 32 & 20.8 & 0.254 & 0.0745 & 0.794 \\
$\mathrm{Si}$ & 3.476 & 0.4459 & 26 & 20.6 & 0.221 & 1.1509 & 0.751 \\
$\operatorname{In}_{\mathrm{x}} \mathrm{Ga}_{1-\mathrm{x}} \mathrm{As}$ & 3.536 & 0.4389 & 5 & 20.6 & 0.213 & $2.01 \mathrm{E}-10$ & 0.740 \\
\hline
\end{tabular}

\section{Saturable absorber behavior of graphene polymeric composite}

In this section, in order to consider the carrier dynamics of graphene/PMMA inside the Maxwell's equation, we have implemented the macroscopic polarization vector as following

$$
\mathrm{D}=\varepsilon_{0} \varepsilon_{h} E+\sum_{m} P_{m}
$$

in which the $\varepsilon_{h}$ is the permitivity of the host material and $P_{m}$ is the polarization induced by the $m^{\text {th }}$ oscillator. Taking into consideration a source term dependent to the difference of the population density in ground state and excited level, it is possible to define the macroscopic polarization by an Axillary Differential Equation (ADE) as follow

$$
\frac{d^{2} P_{10}}{d t^{2}}+\gamma_{10} \frac{d P_{10}}{d t}+\omega_{0}^{2} P_{10}=\kappa\left(N_{0}-N_{1}\right) E_{y}
$$

where $P_{10}$ is the average macroscopic polarization vector, which is a function of time and space, $\gamma_{10}$ is the damping coefficient of this oscillator and the $\omega_{0}$ is the transition frequency. The coupling factor $\kappa$ takes the form of $\kappa=6 \pi \varepsilon_{0} c^{3} /\left(\tau \omega_{0}^{2} \sqrt{\varepsilon_{h}}\right)$. By considering the graphene/PMMA as a two level system, the transition between this two level can be described with an ADE as follow

$$
\frac{d N_{1}}{d t}=-\frac{N_{1}}{\tau}+\frac{1}{\hbar \omega_{0}} E_{y} \cdot \frac{d P}{d t}
$$

here the population density of the excited state and ground state is expressed as $N_{l}$ and $N_{2}$. By adding these equations as two ADE, we can relate the Maxwell's equation to the carrier dynamic of the polymeric composite in saturable absorber part. 


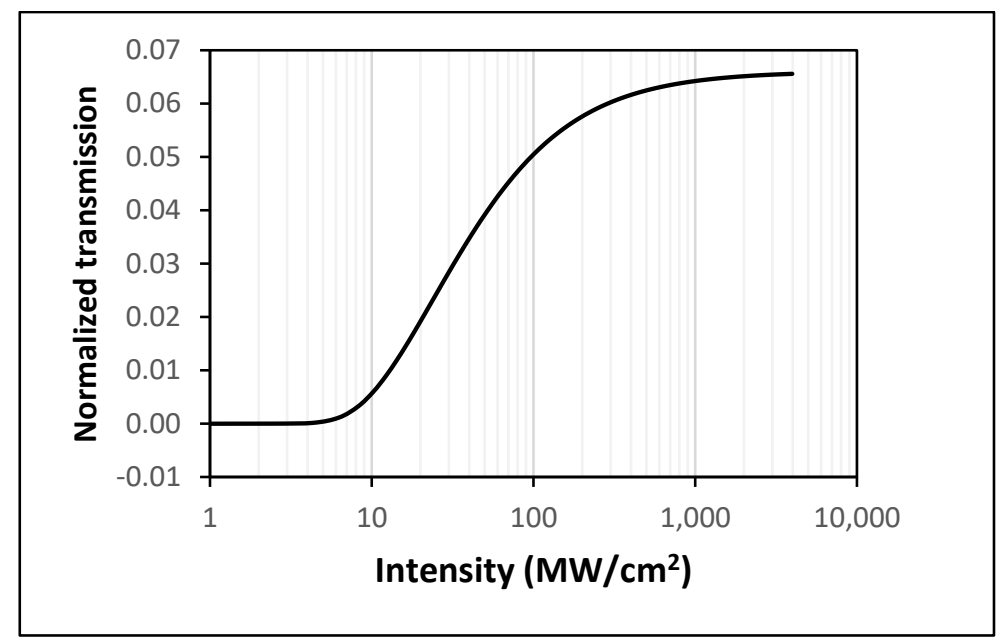

Figure 2: Nonlinear transmission curve as a function of the input port intensity for graphene polymeric composite at $1550 \mathrm{~nm}$ wavelength
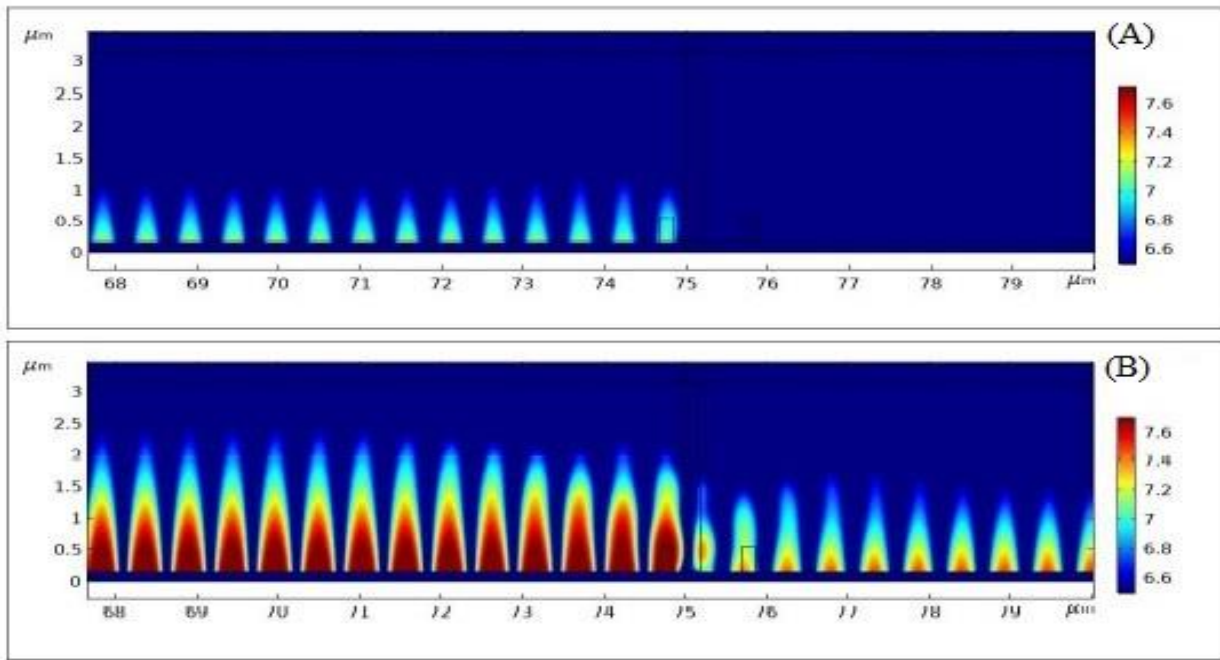

Figure 3: SPP intensity profile (A) before reaching to the saturation intensity and (B) after reaching to the saturation intensity of graphene polymeric composite

As can be seen from the Figure 2, there is an S shape trend in the transmission of SPP which represents the saturation behavior of the absorption coefficients. From this experiment we have found that the saturation intensity is $53.5 \mathrm{MW} / \mathrm{cm}^{2}$ for a layer of SA with $200 \mathrm{~nm}$ thickness. The modulation depth for this structure is about $6 \%$. This nonlinear transmission curve shows a perfect compatibility to the activation curve of sigmoid type which is an essential element in the neuromorphic applications. Figure 3, indicates the two state of the system, (A) before reaching the saturation intensity which occurs in low input power and (B) after reaching the saturation intensity. Since the intensity of SPP is lower in the (A), it completely suppressed by the SA and no field is transmitted, but when the intensity of the SPP passes through the saturation threshold, part of the SPP intensity transmits to the other side of the structure in post synaptic region. In order to further analyze this nonlinear activation function, it has been set up a simulation to further investigate the effect of the thickness of the saturable absorber layer on the saturation intensity and the modulation depth.

\section{Thickness of the saturable absorber layer}

In order to see what is the effect of saturable absorber thickness on the modulation depth and saturation intensity (defined as the SPP intensity required to reduce the absorption to half of its unbleached value), we have studied 
the model in the previous section with different SA thickness, ranging from $100 \mathrm{~nm}$ to $500 \mathrm{~nm}$. The lowest saturation intensity as depicted in occurs for $100 \mathrm{~nm}$ thickness which is about $34.1 \mathrm{MW} / \mathrm{cm} 2$. By increasing the thickness of the SA layer, since the absorption length is increasing, as a result, we will have saturation at higher intensities and the curves shift to the higher values. From the Figure 4, it is clear that the modulation depth for all of the thickness values is nearly the same and lies between 6 and $7 \%$, but for the $300 \mathrm{~nm}$, this value is about $8.5 \%$.

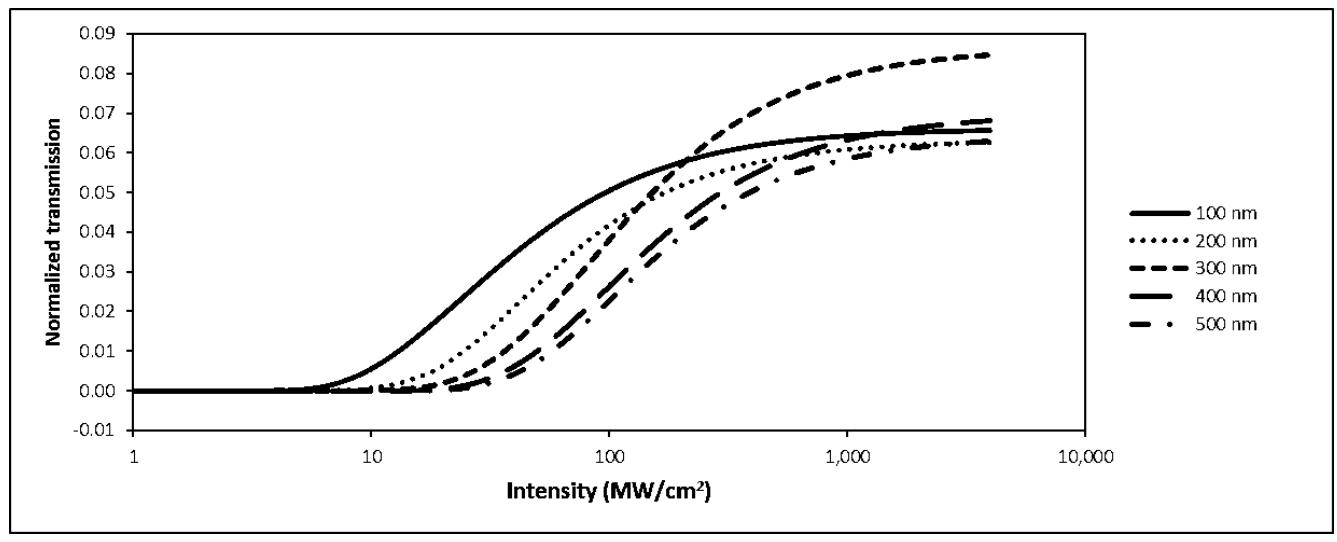

Figure 4: Effect of different thickness of saturable absorber layer on the saturation behavior of the graphene/PMMA composite

Since in this simulation we have a normal incidence of SPP field to the SA layer so we have two conditions for the interference of the back-reflected light as following

$$
\begin{array}{ll}
2 n_{S A} d_{S A}=\left(m-\frac{1}{2}\right) \lambda_{0} & \text { for constructive interference of reflected light } \\
2 n_{S A} d_{S A}=m \lambda_{0} & \text { for destructive interference of reflected light }
\end{array}
$$

So in order to prevent the back reflection of the SPP and increase the transmission to the other side of the structure it is required to have a thickness of the $\mathrm{SA}$ equal to $d_{S A}=\frac{m \lambda}{2 n_{S A}}=\frac{1550 \mathrm{~nm}}{2 \times 2.4793}=312 \mathrm{~nm}$, for $\mathrm{m}=1$. This is the reason why we observed the higher modulation depth for the SA with $300 \mathrm{~nm}$ thickness. Figure 5 (A) indicates further explanation for this observation which represents the reflectance of SPP by sweeping the SA thickness from 100 to $500 \mathrm{~nm}$. The results show a periodic trend with a minimum value of the reflectance around $310 \mathrm{~nm}$ which is compatible with derived value of $312 \mathrm{~nm}$ and a maximum value of reflectance at $460 \mathrm{~nm}$. This result is also represented visually in Figure 5 (B and C) in which the transmission of SPP is reduced because of the high reflection at $460 \mathrm{~nm}$ of thickness. This is the reason why by increasing the thickness of the SA layer we observe a reduction in the modulation depth of $\mathrm{SA}$. 


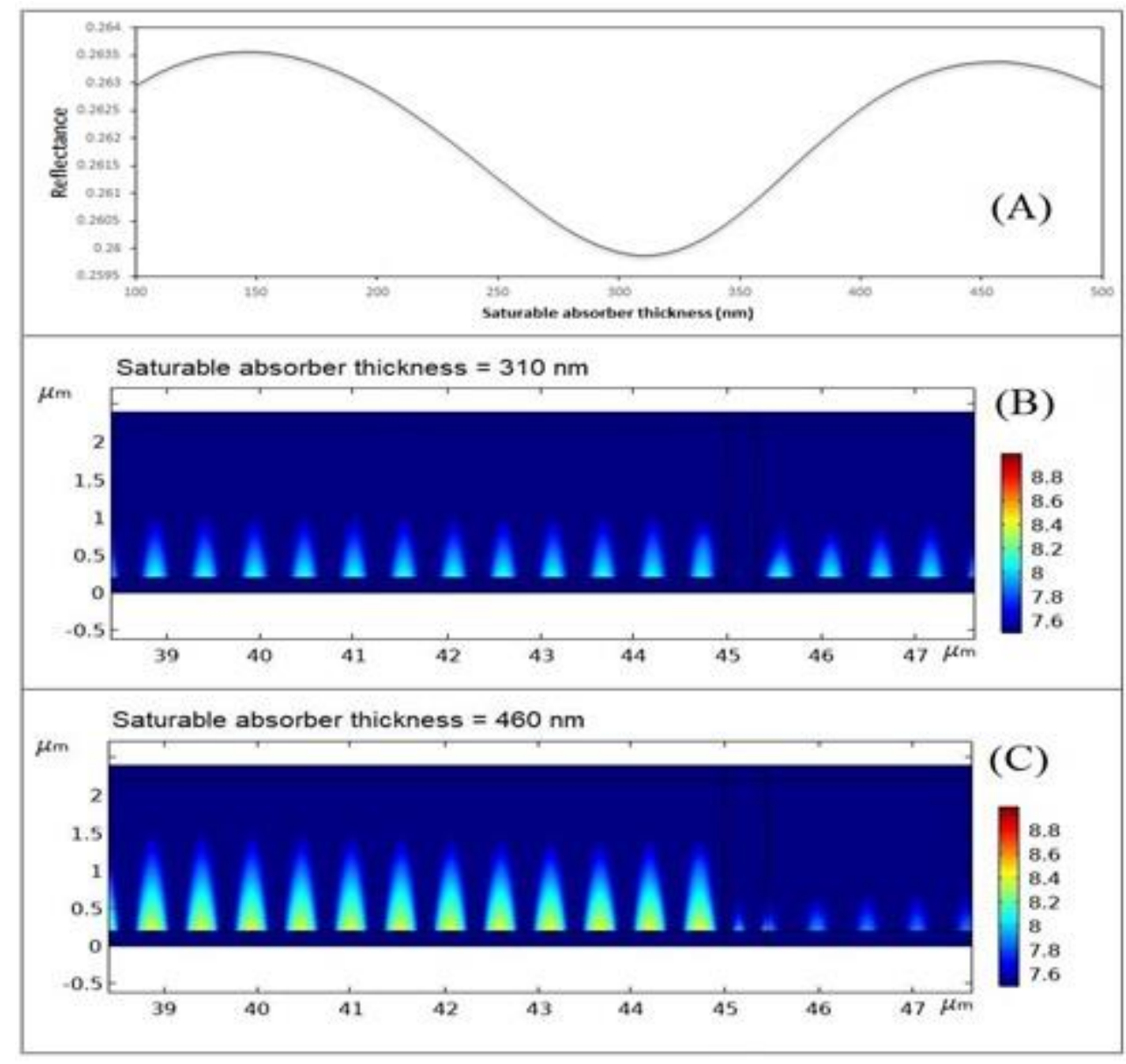

Figure 5: (A) Reflectance of SPP for different values of saturable absorber thickness from $100 \mathrm{~nm}$ to $500 \mathrm{~nm}$, (B) Graphical representation of the SPP reflectance magnitude at minimum values of $310 \mathrm{~nm}$ and (C) maximum at $460 \mathrm{~nm}$.

\section{Conclusion}

Taking into account the results of this study, it is possible to demonstrate a fully functioning all-optical nonlinear activation function, based on the saturable absorber property of graphene/PMMA. The synaptic behavior of this saturable absorber material provides a passive saturable optical response for the SPP signal. All of the optical properties of the graphene have been adopted from the recent experimental works and the results of this simulation are closely consonant with those results. The demonstrated SA scheme can be used to construct various neuromorphic architectures with intrinsic and passive optical computation. The maximum value for the modulation depth is possible for the thickness of $310 \mathrm{~nm}$ and is equal to $8.5 \%$ which compared to the other values of thickness is considerable. This behavior is mainly because of the destructive interference of the back-reflected SPP field which is reflected from the second interference and reduces the reflection coefficient of intensity. As a result, the transmitted SPP intensity is higher in $310 \mathrm{~nm}$ thickness and this trend repeats periodically.

\section{Code Availability}

Not applicable

\section{Data Availability}

The data that support the findings of this study are available from the corresponding author upon reasonable request.

\section{Authors' Contributions}

All authors contributed to the study conception and design. COMSOL numerical analysis and Data collection were performed by Dr. Hamed Tari. COMSOL environment was set up by Dr. Alessandro Bile. Professor Eugenio Fazio provided the workstations and technical assistance during the COMSOL calculations. The first draft of the manuscript was written by Francesca Moratti. 


\section{Funding}

Authors gratefully acknowledge the financial support from Department of Basic and applied Science for Engineering of Sapienza university of Rome (Avvio alla Ricerca, Sapienza University of Rome AR120172B7152382).

\section{Conflicts of interest/Competing interests}

The authors declare that they have no conflict of interest.

\section{References}

1. Rosenbluth D, Kravtsov K, Fok MP, Prucnal PR (2009) A high performance photonic pulse processing device. Optics express 17 (25):22767-22772

2. Lu W, Lieber CM (2006) Semiconductor nanowires. Journal of Physics D: Applied Physics 39 (21):R387

3. Fazio E, Chauvet M, Vlad V, Petris A, Pettazzi F, Coda V, Alonzo M (2009) 3-D integrated optical microcircuits in lithium niobate written by spatial solitons. In: Ferroelectric Crystals for Photonic Applications. Springer, pp 101-134

4. Tong L, Sumetsky M (2009) Subwavelength and Nanometer Diameter Optical Fibers (Zhejiang. Zhejiang University Press, 5. Dumon P, Bogaerts W, Wiaux V, Wouters J, Beckx S, Van Campenhout J, Taillaert D, Luyssaert B, Bienstman P, Van Thourhout D (2004) Low-loss SOI photonic wires and ring resonators fabricated with deep UV lithography. IEEE Photonics Technology Letters 16 (5):1328-1330

6. Maier SA (2007) Plasmonics: fundamentals and applications. Springer Science \& Business Media,

7. Reinhardt C, Passinger S, Chichkov BN, Marquart C, Radko IP, Bozhevolnyi SI (2006) Laser-fabricated dielectric optical components for surface plasmon polaritons. Optics letters 31 (9):1307-1309

8. Avrutsky I, Soref R (2011) Phase-matched sum frequency generation in strained silicon waveguides using their secondorder nonlinear optical susceptibility. Optics express 19 (22):21707-21716

9. Zhang H, Tang D, Knize RJ, Zhao L, Bao Q, Loh KP (2010) Graphene mode locked, wavelength-tunable, dissipative soliton fiber laser. Applied Physics Letters 96 (11):111112

10. Zhang H, Tang D, Zhao L, Bao Q, Loh K (2009) Large energy mode locking of an erbium-doped fiber laser with atomic layer graphene. Optics Express 17 (20):17630-17635 
Figures

(A) GRATING COUPLER

\section{SILVER SPP SUPPORT}

(B)

Figure 1

(A) Schematic representation of the simulated structure (B) Excitation of the structure with a periodic port with intensity before saturation intensity of the saturable absorber and complete suppression of the SPP field.

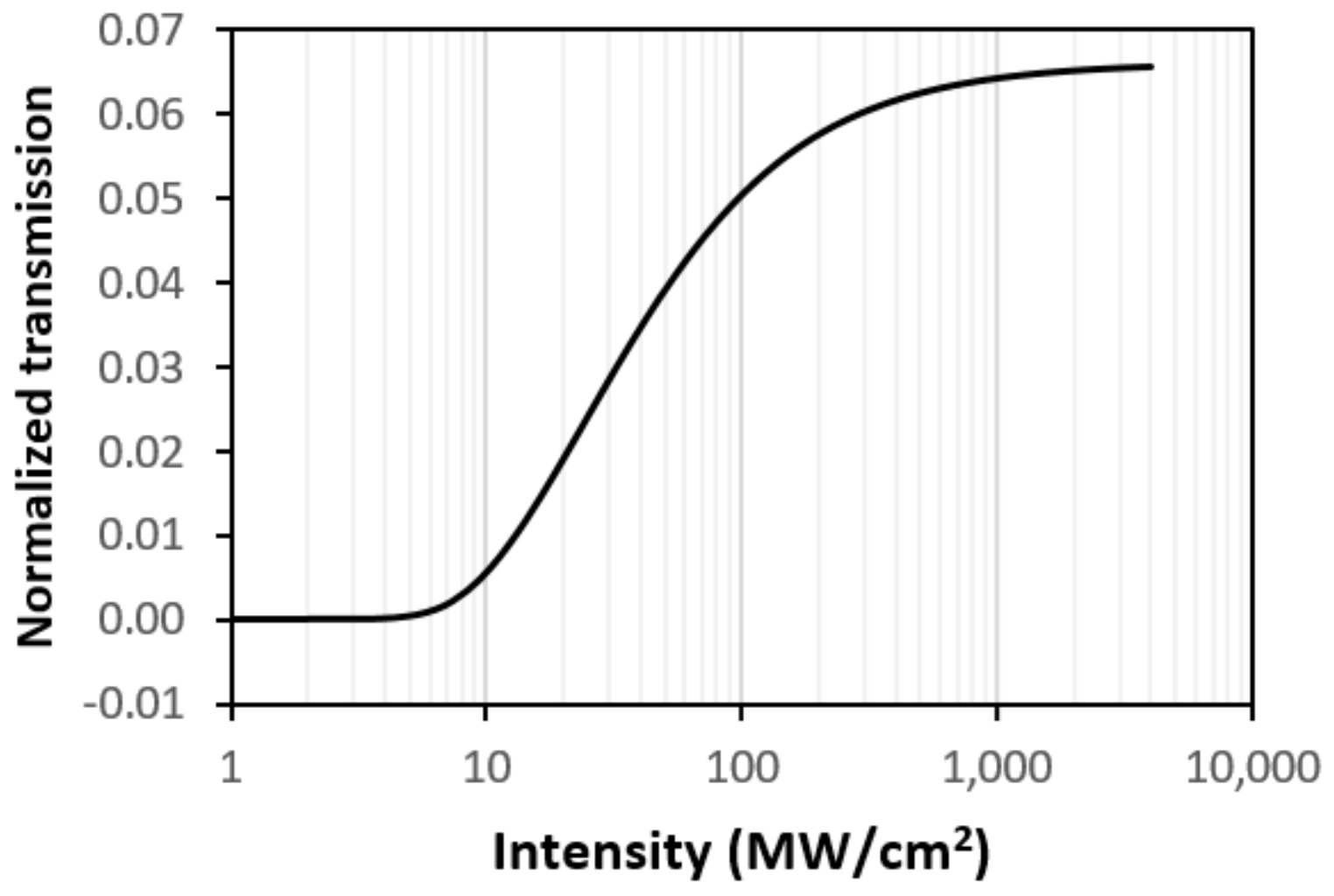

Figure 2 
Nonlinear transmission curve as a function of the input port intensity for graphene polymeric composite at $1550 \mathrm{~nm}$ wavelength
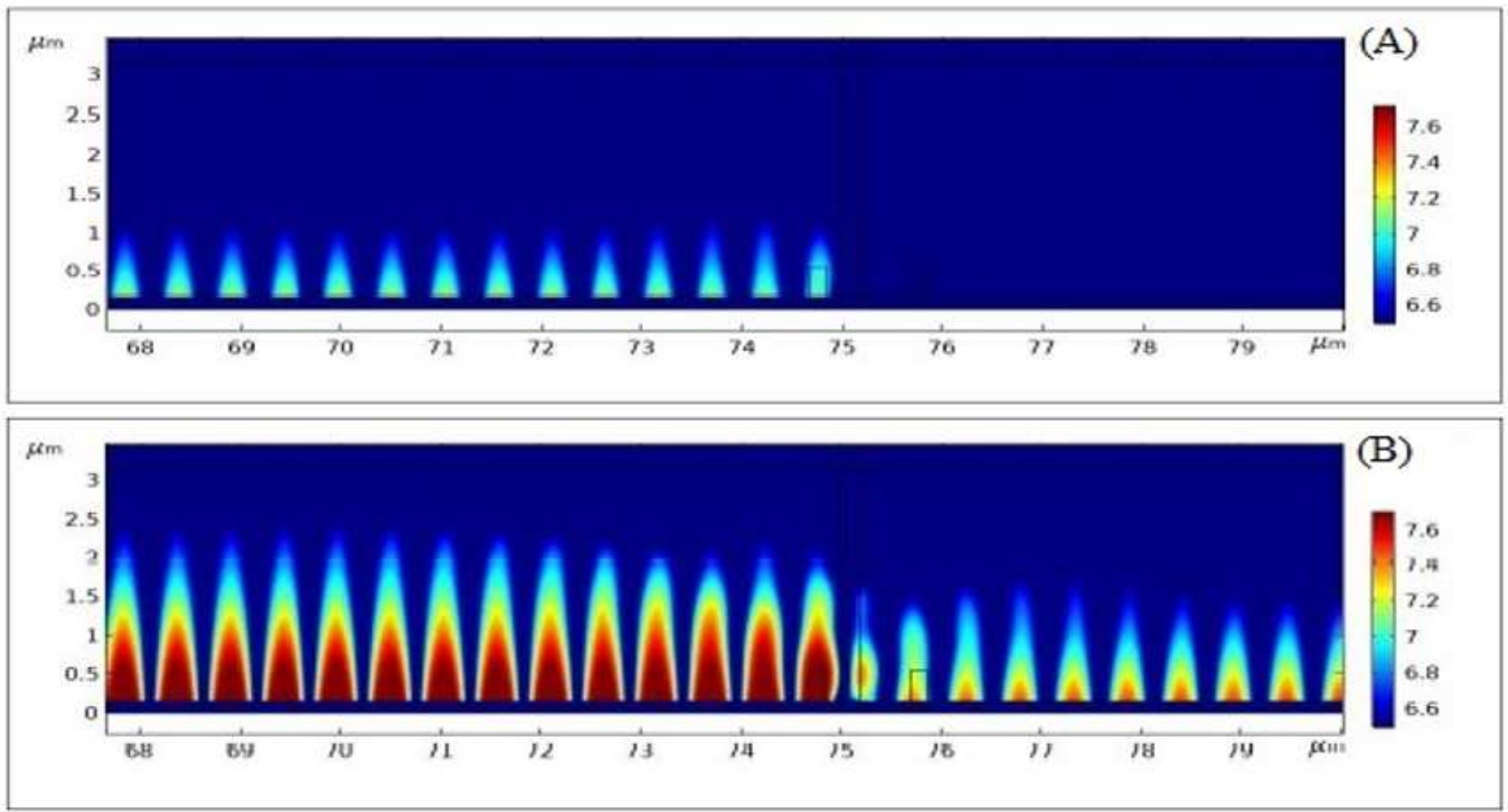

Figure 3

SPP intensity profile (A) before reaching to the saturation intensity and $(B)$ after reaching to the saturation intensity of graphene polymeric composite

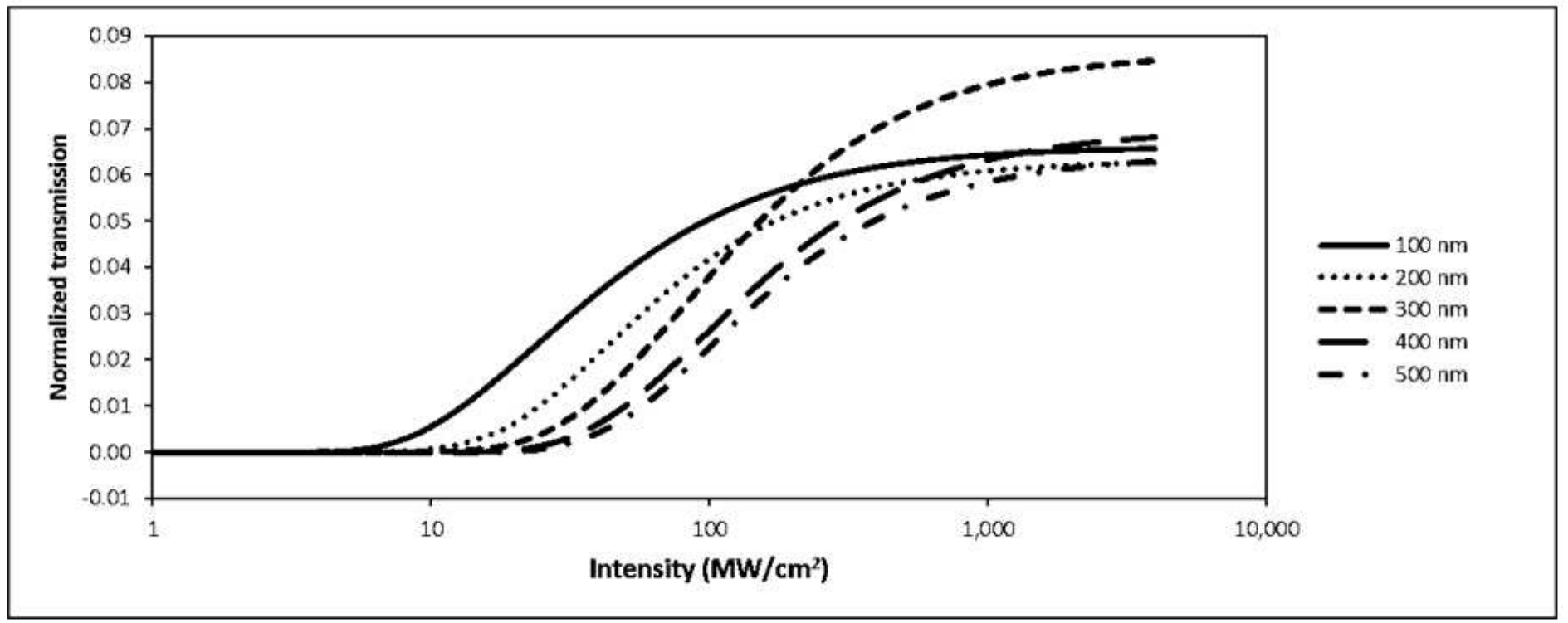

Figure 4 
Effect of different thickness of saturable absorber layer on the saturation behavior of the graphene/PMMA composite

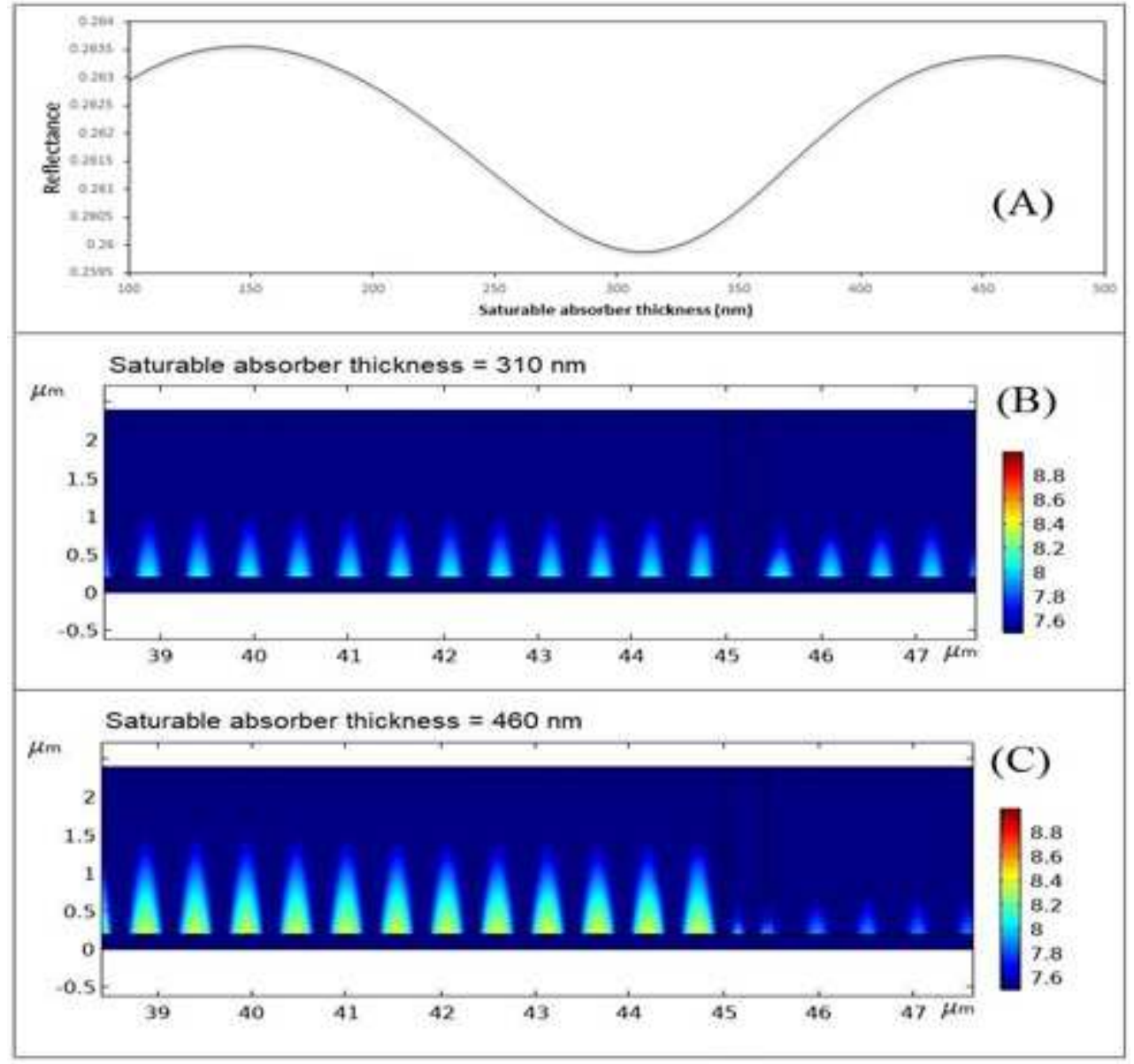

Figure 5

(A) Reflectance of SPP for different values of saturable absorber thickness from $100 \mathrm{~nm}$ to $500 \mathrm{~nm}$, (B) Graphical rep-resentation of the SPP reflectance magnitude at minimum values of $310 \mathrm{~nm}$ and (C) maximum at $460 \mathrm{~nm}$. 